\title{
MAGNETIC LATTICE FOR THE HIF NEUTRALIZED TRANSPORT EXPERIMENT (NTX)*
}

\author{
D. Shuman", S. Eylon, E. Henestroza, P. K. Roy, W. Waldron, S. S. Yu, LBNL, T. Houck, LLNL
}

\section{Abstract}

The NTX experiment at the Heavy Ion Fusion Virtual National Laboratory is exploring the performance of neutralized final focus systems for high perveance heavy ion beams. A pulsed magnetic four-quadrupole transport system for a $400 \mathrm{keV}, 80 \mathrm{~mA}$ space charge dominated heavy ion beam has been designed, fabricated, tested, measured, and commissioned successfully for the Neutralized Transport Experiment (NTX). We present some generalized multipole decompositions of 3-D finite element calculations, and 2-D transient finite element simulations of eddy currents in the beam tube. Beam envelope calculations along the transport line were performed using superposition of individually 3-D calculated magnetic field maps. Revised quadrupole design parameters and features, plus fabrication and testing highlights are also presented. Magnetic field measurements were made using both Hall probes (low field DC) and inductive loop coil (high field pulsed). Magnet testing consisted of repetitive full current pulsing to determine reliability.

\section{MAGNETIC LATTICE}

The transport section is designed to correspond closely to a prototypical HIF driver final focus channel. It consists of a double FODO channel with very short drift regions, including the drift from the source into the channel, and the drift into the plasma neutralization chamber. Fig. 1 shows the $\mathrm{X}$ and $\mathrm{Y}$ beam profiles through the system, with and without beam neutralization at the exit. The beam is quite large in the two center magnets, which determines the required bore size and winding radius. The magnet has a relatively short center section and a substantial portion of the magnetic field is contained in the end fringe fields, with significant axial components. The heavy ion beam is space charge dominated, having a large cross section, and being highly non-paraxial. As such, the usual method of (beam axis integrated) field quantification into normal multipoles of discrete length does not allow accurate particle tracking simulation, as these hard-edge field simplifications do not contain the axial field components and nonlinear (with radius) gradients which are a significant source of particle deflection. Thus, both normal and pseudomultipoles are important and both must be considered in lattice design, requiring 3D magnetic analysis.

\footnotetext{
"DBShuman@1bl.gov

*This work has been performed under the auspices of the US DOE by UC-LBNL under contract DE-AC03-76SF00098, for the Heavy Ion Fusion Virtual National Laboratory.
}

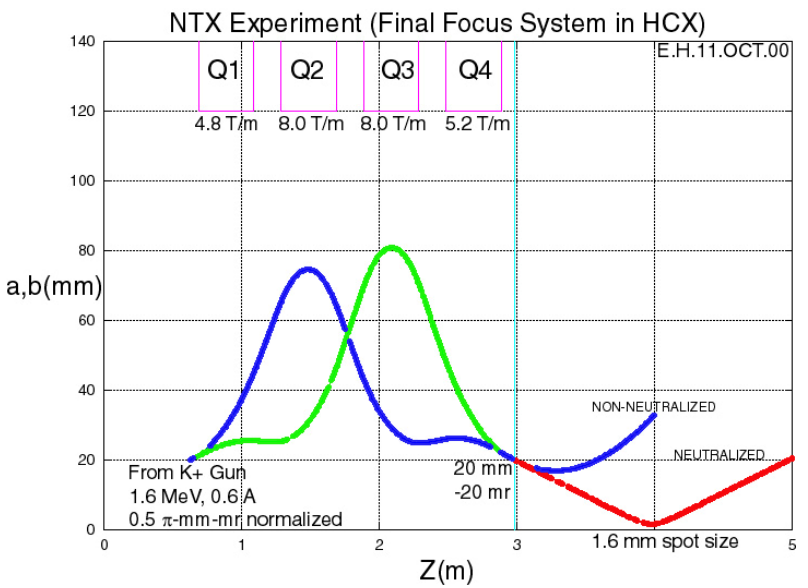

Figure 1. Magnetic Lattice

\section{MAGNETIC FIELD MODELING}

A three-dimensional finite element model of the magnet was generated, using ANSYS/EMAG and solved for the static field in 3D space. A scalar potential formulation was used, with source current elements used for the coil. The space modeled was a $1 / 8^{\text {th }}$ section of one half the magnet. Far field (infinite boundary) elements bounded the outer radius and end, at a distance $75 \mathrm{~cm}$ from the magnet midplane, which extends well past the source and final focus points. A cylindrical Neumann boundary was used to simulate the steel core, in order to speed analysis for a fine mesh model. An earlier coarse mesh model with steel included (nonlinear B-H curve) showed no significant saturation.

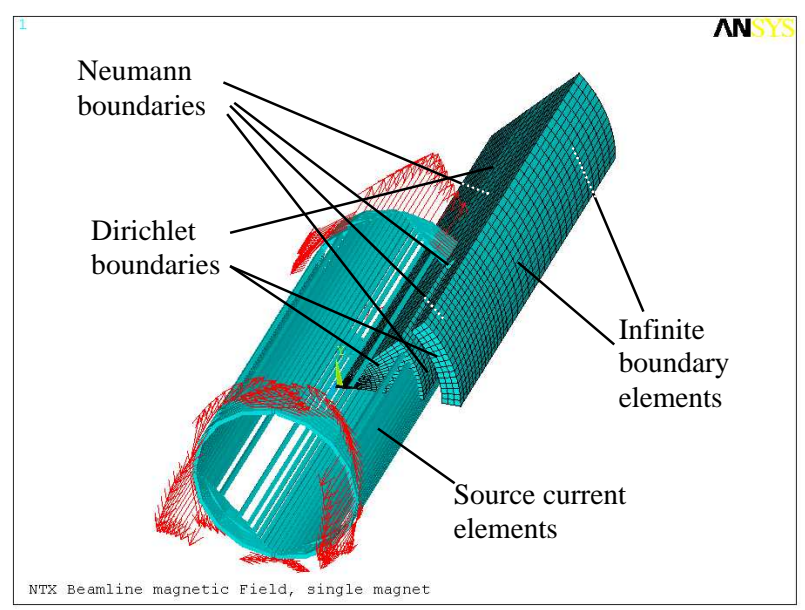

Figure 2. Symmetric Magnet Model, Half Octant

The resulting 3D field maps generated were decomposed into both normal and pseudomultipoles as a function of distance in the axial direction. Figure 3 shows these 
multipoles as a function of distance in Z. Both half octant models and full magnet f.e. models, complete with spiral coil geometry and leads, were computed to determine both symmetry-allowed and full skew (normal and skew) multipoles, respectively. These are the dominant field quantities for this short but highly non-paraxial beam, with higher order normal multipoles being relatively unimportant. Finally, beam tracking simulation through a lattice of focusing elements generated by multiple superposition of these field maps was performed.

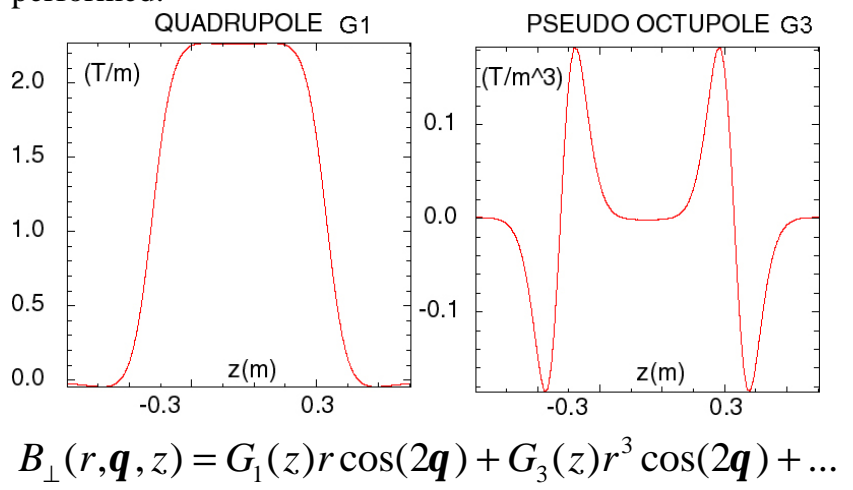

Fig. 3. Dominant Multipoles

\section{MAGNET DESIGN, FAB., \&TEST}

\section{Coil Design}

The magnet design concept is essentially unchanged from the design presented at PAC2001 ${ }^{1}$. Subsequent changes include a larger bore and winding radius, plus a simplified coil design. Revised design and operating parameters are shown in table 1 below:

\begin{tabular}{|c|c|c|}
\hline Beam Aperture Radius, $\mathrm{R}_{\mathrm{b}}$ & 14.9 & $\mathrm{~cm}$ \\
\hline Magnet Winding Radius, $\mathrm{R}_{\mathrm{W}}$ & 17.32 & $\mathrm{~cm}$ \\
\hline Steel Inner Radius, $\mathrm{R}_{\mathrm{W}}$ & 18.33 & $\mathrm{~cm}$ \\
\hline Steel Outer Radius, $\mathrm{R}_{\mathrm{O}}$ & 25.63 & $\mathrm{~cm}$ \\
\hline Mag., Total Lengths, $\mathrm{L}_{\mathrm{m}}, \mathrm{L}_{\mathrm{o}}$ & 46,50 & $\mathrm{~cm}$ \\
\hline Magnet to magnet spacing & 60 & $\mathrm{~cm}$ (ctr.-ctr.) \\
\hline Field Gradient, B' & $2-5$ & $\mathrm{~T} / \mathrm{m}$ \\
\hline Maximum Field, B & 0.6 & $\mathrm{~T}, @ 12 \mathrm{~cm}$ \\
\hline Number of turns, $\mathrm{N}$ & 8 & Turns/coil \\
\hline $\begin{array}{c}\text { 2D Field Coefficients, } B_{n} \\
\left(\sum n\left|A_{n}\right| / 2 A_{2}, n=6,10, \ldots, 26\right)\end{array}$ & $7 \times 10^{-4}$ & T/T @10cm \\
\hline Conductor diameter, $\mathrm{d}_{\mathrm{c}}$ & 4.65 & $\mathrm{~mm}$ \\
\hline Magnet Current, $I_{\min ,}-I_{\max }$ & $3.3-8.2$ & $\mathrm{kA}$ \\
\hline Magnet Resistance, $\mathrm{R}$ & .036 & $\Omega$ \\
\hline Magnet Inductance, L & 232 & $\mu \mathrm{H}$ \\
\hline Pulse length (full half sine), $\mathrm{t}$ & 2.2 & $\mathrm{mS}$ \\
\hline Magnet Voltage, max., V & 2.7 & $\mathrm{kV}$ \\
\hline Pulse energy, max., U & 7.8 & $\mathrm{~kJ}$ \\
\hline Energy loss/pulse, max., $\mathrm{Q}_{\mathrm{t}}$ & 2.7 & $\mathrm{~kJ}$ \\
\hline Max., Operating Pulse Rates & $0.5,0.1$ & $\mathrm{~Hz}$ \\
\hline Temp. Rise, Max., steady state & 25 & ${ }^{\circ} \mathrm{C},(0.5 \mathrm{~Hz}$ P.R $)$ \\
\hline
\end{tabular}

Table 1. Revised Magnet Parameters

\section{Fabrication}

The magnet was fabricated as per ref. 1, with the following improvements:

- To simplify coil fabrication, a simplified coil design featuring a novel diagonal leadout was used. The diagonal leadout (from the inner turn) approximates a "stairway" of "missing" currents that are inherently present in any spiral coil. This allows short coils with few turns to have relatively high field symmetry. A concept is shown below in fig.3.

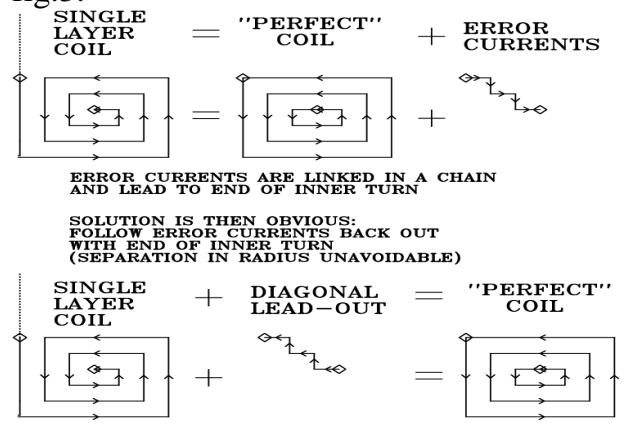

Figure 3. Diagonal Coil Leadout Concept

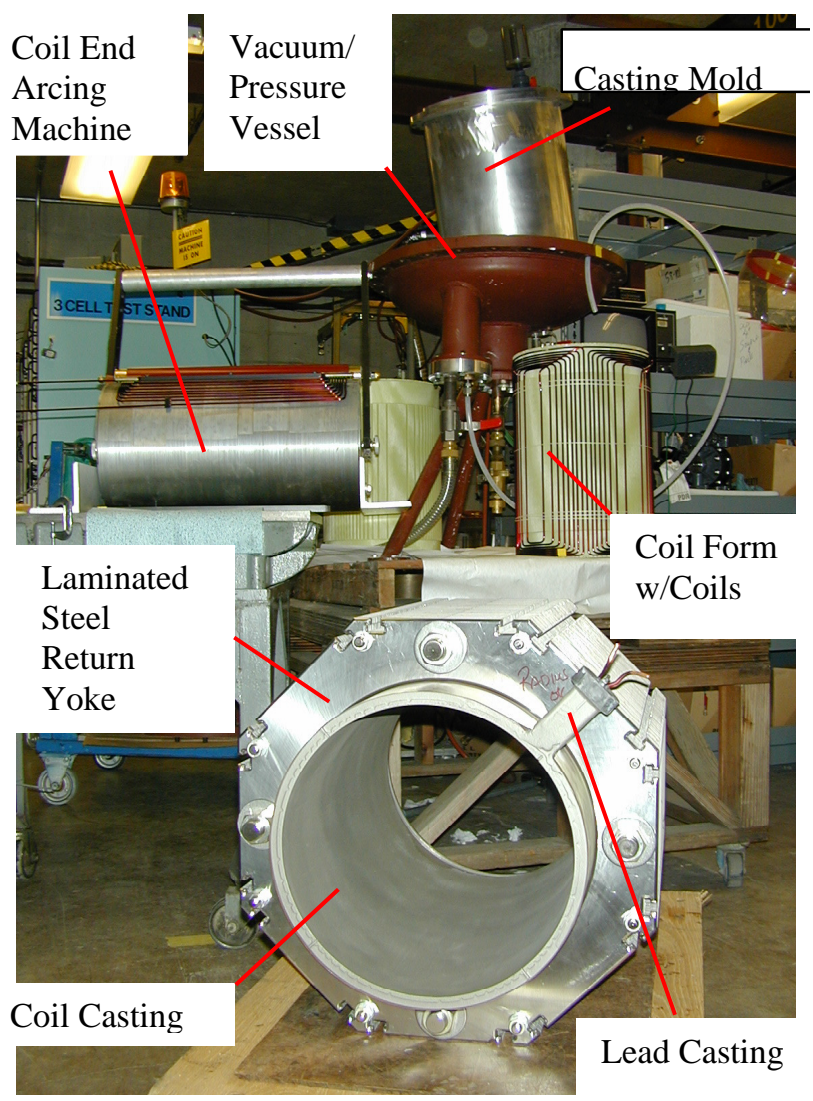

Fig. 4 Magnet Fabrication

- A lead casting section was added to provide an exit for the leads that was readily accessible on the exterior of the core for repairablity. This section was designed to flare the ground surface (provided 
by resistive paint) away from the leads where they exit the casting to avoid high field concentrations. This enables the leads to be kept close together in the coil area near the beam, minimizing field errors from them.

\section{Operation}

- A $2 \mathrm{mF}$ pulser capacitance was chosen in order to increase the pulse length, reducing voltage and eddy currents.

\section{Testing}

- Six quadrupoles total were fabricated, to provide two spares, one of which was subjected to 10,000 full current pulses for testing reliability. All other magnets were subjected to 1,000 full current pulses for acceptance testing. No cooling, other than free air convection is necessary.

- Field was measured with a system of 12 high impedance inductive pickup coils mounted to a four arm "cross", with three three orthogonal coils mounted on each arm end. Primary goals were find the magnetic center and azimuthal axes for each magnet, though gross multipole measurements were possible. The coils are open circuit and induced voltage is measured and integrated over the magnet pulse. Figure 5 shows the axial field falloff profile compared with the computed model (peak fields normalized for comparison).

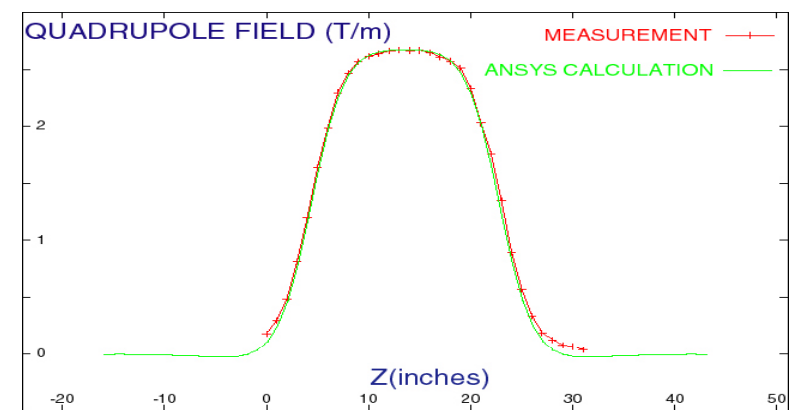

Figure 5. Calculated vs. Measured Gradient

\section{BEAM TUBE EDDY CURRENTS}

Both 2D and 3D transient magnetic modeling was performed to determine the effect of eddy currents in surrounding components such as beam tubes, flanges, diagnostics, electron traps, etc. Per ref. 1, coil and core eddy currents were calculated to be insignificant. The thin-walled stainless steel/fiberglass composite beam tube specified in ref. 1 was manufactured by an outside vendor, but the S.S. liner subsequently delaminated immediately after cure. The vendor used air pressure to hold the S.S. tube round while filament winding over it. It is thought that insufficient care was taken to balance the tensile hoop (and possibly longitudinal) stresses from air pressure against hoop compressive stresses generated from winding, to avoid residual tensile stresses in the liner. A 0.134" wall thickness standard 12" dia. S.S. vacuum tube was used instead. Eddy currents were calculated by transient 2-D finite element analysis and found to be acceptable, with a $7.4 \%$ loss of peak field, and a $0.28 \mathrm{mS}$ field peak time lag from the source current peak, as shown in figures 6 and 7.

The close magnet spacing precluded the use of core steel overhang on the coils. Such overhang, normally used to clamp off end fields, would act to couple the quadrupoles via their end fields, making them difficult to adjust and increasing eddy currents in the end laminations and plates. Similarly, vacuum flanges located in the high field regions or between the magnets could be susceptible to high eddy currents, as well.

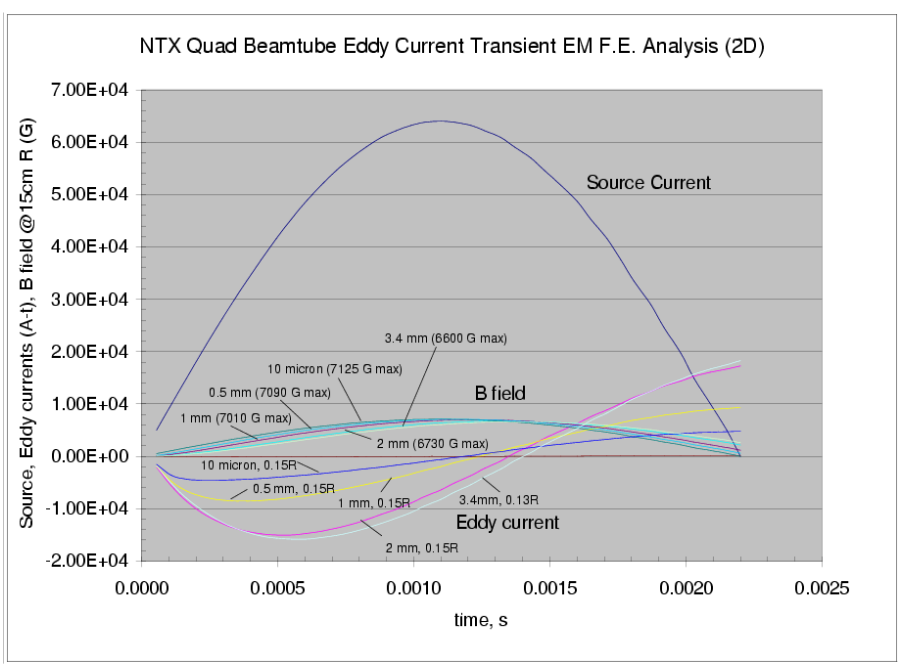

Figure 6: Beam tube Eddy Currents and B fields.

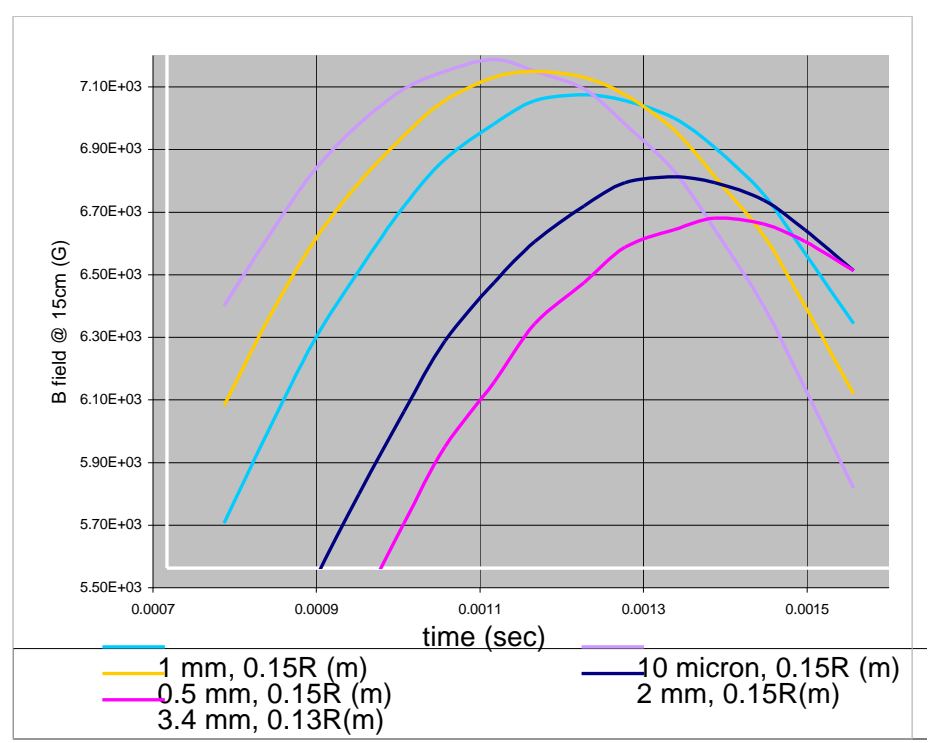

Figure 7: Peak B field vs. beam tube thickness and radius.

\section{REFERENCES}

[1] A Large Bore Pulsed Quadrupole Magnet for Transport of High Current Beams at Low Energies, D. Shuman, et. al., PAC2001, pg. 2937 